Pacific Journal of Mathematics

CONCERNING PARTIAL RECURSIVE SIMILARITY

LoUISE HAY, ALFRED BERRY MANASTE
AND Joseph GoEFFrey Rosenstein 


\title{
CONCERNING PARTIAL RECURSIVE SIMILARITY TRANSFORMATIONS OF LINEARLY ORDERED SETS
}

\author{
Louise Hay, Alfred B. Manaster and \\ JoSEPH G. ROSENSTEIN
}

\begin{abstract}
Interesting contrasts between uncountable suborderings of the continuum and denumerable linear orderings are provided by results of Dushnik and Miller and Sierpiński on the one hand and Laver on other. We investigate analogues of these results in a recursive setting where the only similarity maps are restrictions of partial recursive functions. Complements of recursively enumerable bi-dense subsets of the rationals of arbitrary nonzero degree of unsolvability are shown to bear a strong resemblance to uncountable suborderings of the continuum.
\end{abstract}

1. Introduction and summary. Two linear orderings, $H$ and $G$, are said to be similar if there is an order preserving map from $H$ onto $G$. $H$ is said to be embeddable in $G$ if $H$ is similar to a subordering of $G$. $H \leqq G$ denotes that $H$ is embeddable in $G$ while $H \$ G$ denotes that $H$. is not embeddable in $G$. $c$ denotes the cardinality of the continuum $C$. $\$ denotes the least ordinal whose cardinality is c. $A$ is said to be $b i$-dense in $B$ if both $A$ and its complement in $B$ are dense in $B$.

Theorems I and II below are due to Dushnik and Miller [1940]. Sierpiński [1950] contains Theorems III, IV, and V, whose proofs are interesting elaborations of the techniques and proofs of Dushnik and Miller.

THEOREM I. Every countable linear ordering is similar to proper subset of itself.

THEOREM II. There is a bi-dense subset $A$ of $C$ of cardinality c such that there is no order-preserving map from $A$ into itself except the identity; in particular, $A$ is not similar to any proper subset of itself.

THEOREM III. There is a sequence $\left\{G_{\alpha} \mid \alpha<\boldsymbol{\$}\right\}$ of bi-dense subsets of $C$ such that each $G_{\alpha}$ has cardinality $c$ and $\alpha<\beta<\aleph$ implies $G_{\alpha} \leqq G_{\beta}$ but $G_{\beta} \leqq G_{\alpha}$.

TheOREM IV. There is a sequence $\left\{D_{\alpha} \mid \alpha<\aleph\right\}$ of bi-dense subsets 
of $C$ such that each $D_{\alpha}$ has cardinality $c$ and $\alpha<\beta<\boldsymbol{K}$ implies $D_{\beta} \leqq D_{\alpha}$ but $D_{\alpha} \leqq D_{\beta}$.

THEOREM V. There is a sequence $\left\{H_{\alpha} \mid \alpha<\boldsymbol{\aleph}\right\}$ of bi-dense subsets of $C$ such that each $H_{\alpha}$ has cardinality $c$ and $\alpha<\beta<\boldsymbol{N}$ implies both $H_{\alpha} \geqq H_{\beta}$ and $H_{\beta} \geqq H_{\alpha}$.

In contrast to these results, Laver published in 1971 a proof of the following conjecture of Fraïsse about countable linear orderings.

THEOREM VI. Every descending sequence of countable order types is finite, and every anti-chain (under embeddability) of countable order types is finite.

Theorems I and VI concerning countable order types contrast strongly with Theorems II-V concerning uncountable order types. From a recursion-theoretic point of view, complements of recursively enumerable sets are analogous to uncountable sets, since they are the simplest sets which cannot be effectively enumerated. We shall see that, in fact, co-recursively enumerable orderings (under effective embeddability) bear a strong resemblance to uncountable orderings (under embeddability).

Let us fix an effective $1-1$ correspondence between the natural numbers $N$ and the rational numbers $Q$. (For technical reasons, which will become apparent later, we will assume that those rationals corresponding to natural numbers of the form $4 j$ and $4 j+1$ are dense in $Q$.) We identify each natural number with its corresponding rational number quite freely. $<$ and $\leqq$ refer to the usual ordering of the natural numbers while $(\&$ and $\triangleq$ refer to the usual ordering of the rational numbers. $\phi$ is said to embed $\alpha$ into $\beta$ if $\phi$ is a partial function whose domain includes $\alpha, \varphi(x) \in \beta$ for all $x \in \alpha$, and whenever $x \in \alpha, y \in \alpha$, and $x \otimes y$, then $\phi(x) \ominus \phi(y)$. If $\phi$ embeds $\alpha$ into $\beta$ and $\phi$ is partial recursive, $\phi$ is said to recursively embed $\alpha$ into $\beta$. $\alpha \leqq_{c}^{\varphi} \beta$ denotes that $\varphi$ recursively embeds $\alpha$ into $\beta$. $\alpha \leqq_{c} \beta$ denotes that $\alpha$ is recursively embeddable into $\beta$.

In the next section we present a recursive analogue of the construction used to prove Theorem II. This leads to the results below which are proved in $\S 3$.

THEOREM 2. There is a co-recursively enumerable bi-dense subset $\alpha$ of $Q$ such that there is no order-preserving partial-recursive map from $\alpha$ into itself except extensions of the identity on $\alpha$.

THEOREM 3. There is a sequence $\left\{\gamma_{i} \mid i \in N\right\}$ of co-recursively 
enumerable bi-dense subsets of $Q$ such that $i<j$ implies $\gamma_{i} \leqq_{c} \gamma_{j}$ but $\gamma_{j} \$_{c} \gamma_{i}$.

THEOREM 4. There is sequence $\left\{\delta_{i} \mid i \in N\right\}$ of co-recursively enumerable bi-dense subsets of $Q$ such that $i<j$ implies $\delta_{j} \leqq_{c} \delta_{i}$ but $\delta_{i} \$_{c} \delta_{j}$.

THEOREM 5. There is a sequence $\left\{\eta_{i} \mid i \in N\right\}$ of co-recursively enumerable bi-dense subsets of $Q$ such that $i<j$ implies both $\eta_{i} \oiint_{c} \eta_{j}$ and $\eta_{j} \not \eta_{0} \eta_{i}$.

Furthermore, assume that $\beta$ is any fixed recursively enumerable set which is not recursive. Then the sets $\alpha$ and $\gamma_{\imath}, \delta_{i}, \beta_{i}$ for $i \in N$ can be constructed to be Turing equivalent to $\beta$.

Thus, under recursive embeddability, the co-recursively enumerable bi-dense subsets of $Q$ (of any fixed nonzero degree of unsolvability) bear a strong resemblance to the bi-dense subsets of $C$, but not to the bi-dense subsets of $Q$, under ordinary embeddability.

The following more general consequence of the basic Theorem 1 was proved in response to a question of $R$. Laver. (The brief proofs of Theorems 3, 4, and 5 have been retained to highlight their similarity to the combinatorial proofs of Theorems III, IV, and V.)

THEOREM 7. Let $\beta$ be any recursively enumerable set which is not recursive and let $L$ be any countable linear ordering. Then there is a collection of co-recursively enumerable sets, each of which is bi-dense in $Q$, each of which is Turing equivalent to $\beta$, and such that the collection is linearly ordered by $\leqq_{c}$, and with respect to $\leqq_{c}$ is order-isomorphic to $L$.

Laver also asked whether a similar result was correct for countable partial orderings. In this connection we prove the following theorem.

THEOREM 8. Let $\beta$ be any recursively enumerable set which is not recursive and let $K$ be any finite partial ordering. Then there is a collection of co-recursively enumerable sets, each bi-dense in $Q$, each Turing equivalent to $\beta$, such that, under $\leqq_{c}$, this collection is order-isomorphic to $K$.

The proofs of these results are contained in $\S 4$.

J. Remmel has now completely answered Laver's question by showing that Theorem 8 is correct for an arbitrary countable partial ordering. His proof is an application of the techniques developed 
by Sacks to prove that every countable partial ordering can be embedded in the upper semi-lattice of degrees of recursively enumerable sets.

\section{The basic construction.}

THEOREM 1. Let $\beta$ be any recursively enumerable set which is not recursive. There exists a co-recursively enumerable set $\alpha$ such that

(A) $\alpha$ is bi-dense in $Q$,

(B) $\alpha$ is Turing equivalent to $\beta$, and

(C) if $\alpha \leqq_{c}^{\varphi} \alpha$, then $\varphi(\alpha)=\alpha$ for all $\alpha \in \alpha$.

Proof. The proof is an effectivization of Dushnik and Miller's proof of Theorem II using Yates' "permitting" technique to control the degree of $\alpha$. Before turning to the proof, let us make several observations about the result. The requirement that $\alpha$ be dense rules out the possibility of obtaining a recursively enumerable $\alpha$ satisfying (C). This is easily seen by applying the famous technique of Cantor which shows the categoricity of countable dense linear orderings without end points. If we drop the requirement that $\alpha$ be dense, it is possible to find infinite recursive subsets of the rationals such that if $\alpha \leqq_{c}^{\varphi} \alpha$ then $\varphi(\alpha)=\alpha$ for all $\alpha \in \alpha$. The density of $\alpha$ in Theorem 1 is motivated by its analogy with the density condition in Theorems II-V.

Condition (C) can be viewed as an infinite sequence of requirements on $\alpha$, the $e$ th requirement being that if $\varphi_{e}$ maps $\alpha$ into itself, then $\varphi_{e}$ should be the identity on $\alpha$. The basic Dushnik-Miller strategy is to satisfy the $e$ th requirement by finding a $p_{e}$ in the domain of $\varphi_{e}$ such that $p_{e} \neq \varphi_{e}\left(p_{e}\right)$, and then placing $p_{e}$ into $\alpha$ while placing $\varphi_{e}\left(p_{e}\right)$ into the complement $\bar{\alpha}$ of $\alpha$. Of course the various requirements would lead to conflicts, and such a simple-minded scheme would produce a recursive $\alpha$ which we know is impossible. The standard technique for avoiding such pitfalls is the priority method where, in our situation, the smaller $e$ is, the higher the priority we assign to satisfying the eth requirement. Thus, during the construction, when we find a $p_{e}$ which we may use to satisfy the eth requirement, we irrevocably place $\varphi_{e}\left(p_{e}\right)$ into $\bar{\alpha}$ (since it is to be recursively enumerable), but we can only place $p_{e}$ into $\alpha$ tentatively since we may later discover that $p_{e}=\varphi_{e^{*}}\left(p^{*}\right)$ for some $e^{*}<e$ and then be obliged to put $p_{e}$ into $\bar{\alpha}$ since the $e^{*}$ th requirement has higher priority than the eth requirement.

Condition (A) can also be viewed as an infinite sequence of require- 
ments. Let $I_{0}, I_{1}, I_{2}, \cdots$ be a recursive enumeration of all open intervals of $Q$ with rational endpoints. In order to make $\alpha$ dense in $Q$ it suffices to put into $\alpha$, for each $j$, an element $i_{j}$ of $I_{j}$. Doing so is of course a simple matter, except that, for reasons given above, it may later be necessary to put $i_{j}$ into $\bar{\alpha}$; we will only do so, however, in order to satisfy the $e$ th requirement if $e \leqq j$. It will then be clear, as in all "finite injury" priority arguments, that each requirement is satisfied at most finitely often, and that given any requirement, once all higher priority requirements have been satisfied for the last time, it will be satisfied at most one more time, and, if it is subsequently satisfied, it will remain satisfied.

To make (B) correct, we modify the construction above by simultaneously constructing a sequence $c_{0}, c_{1}, c_{2}, \ldots$ of numbers which will record into $\alpha$ information about $\beta$. Specifically, $c_{j}$ will be in $\alpha$ precisely if $j \notin \beta$, so that $\beta$ will be recursive in $\alpha$, since the sequence $\left\{c_{j}\right\}$ will also be recursive in $\alpha$. (For technical reasons, we will also make particular use of the numbers $\left\{d_{i} \mid i \in N\right\}$ where $d_{i}=c_{i}+1$ for each $i$.) On the other hand, the construction of $\alpha$ will be regulated by a fixed $1-1$ recursive enumeration $b_{0}, b_{1}, b_{2}, \cdots$ of $\beta$, so that oracular knowledge of $\beta$ would give complete information about $\alpha$, and thus $\alpha$ will be recursive in $\beta$. Specifically, at stage $s$ of the construction, a requirement may be satisfied only if its relevant parameters are larger than $b_{s}$ - so that the unexpected enumeration of a small element of $\beta$ will "permit" otherwise forbidden activity.

Since each $p_{j}, i_{j}, c_{j}$ and $d_{j}$ may have different values at different stages of the construction, we introduce markers in the style of Rogers. These markers, denoted $p_{j}, i_{j}, c_{j}$ and $d_{j}$ respectively, will be assigned values at certain stages of the construction. The final positions of these markers will be numbers $p_{j}, i_{j}, c_{j}$ and $d_{j}$ respectively, which will fulfill the roles assigned to them in the discussion above.

We assume that, at the end of stage $s$ of the construction, the markers

$$
\left\{p_{j}, i_{j} \mid 0 \leqq j \leqq s\right\} \cup\left\{c_{j}, d_{j} \mid j \in N\right\}
$$

have been assigned the values

$$
M^{s}=\left\{p_{j}^{s}, i_{j}^{s} \mid 0 \leqq j \leqq s\right\} \cup\left\{c_{j}^{s}, d_{j}^{s} \mid j \in N\right\}
$$

respectively. These values are all distinct, except that various $p_{j}^{q}$ may equal 0 . We also assume that $d_{j}^{s}=c_{j}^{s}+1>j+2$ for all $j$, and that, initially, $d_{j}^{0}=4 j+3$ for all $j$. We let

$$
M_{e}^{s}=\left\{p_{j}^{s}, i_{j}^{s}, c_{j}^{s}, d_{j}^{s} \mid j<e\right\} .
$$

We further assume that no $p_{j}^{s}$ or $i_{j}^{s}$ is in $\bar{\alpha}^{s}$, which is the finite subset 
of $\bar{\alpha}$ enumerated by the end of stage $s$; and that $c_{j}^{s}$ or $d_{j}^{s}$ is in $\bar{\alpha}^{s}$ only if $j=b_{t}$ for some $t<s$, in which case both are in $\bar{\alpha}^{s}$.

At stage $s+1, e$ is said to require attention if $e \leqq s, p_{e}^{s}=0$, and there is an $x$ such that $b_{s+1} \leqq x<\varphi_{e}^{s}(x)$, which is defined, such that $x \notin \bar{\alpha}^{s} \cup M_{e}^{s}$ and $\varphi_{e}^{s}(x) \notin M_{e}^{s}$, and with $b_{s+1}=x$ only if $x=c_{j}^{s}$ for some $j \geqq e$. The number $y$ is said to be free at a substage of stage $s+1$ if $y>b_{s}+1, y$ has not been enumerated in $\bar{\alpha}$, and $y$ has never been the value of any marker.

If no $e$ requires attention at stage $s+1$, the only action to be taken at stage $s+1$ is that specified in the next paragraph. Otherwise let $e$ be the last number requiring attention at stage $s+1$ and let $x$ be the least number appropriate for $e$ in the sense of the preceding paragraph. Let $p_{e}^{s+1}=x$ and place $\varphi_{e}(x)$ into $\bar{\alpha}$. If any $p_{j}^{s} \in\left\{x, \varphi_{e}(x)\right\}$, for each such $j$ let $p_{j}^{s+1}=0$. If any $i_{j}^{s} \in\left\{x, \varphi_{e}(x)\right\}$, for each such $j$, in order, let $i_{j}^{s+1}$ be the least free number in $I_{j}$. Consider, in order, those $j$ for which $\left\{c_{j}^{s}, d_{j}^{s}\right\} \cap\left\{x, \varphi_{e}(x)\right\} \neq \varnothing$ : if $\varphi_{e}(x) \in\left\{c_{j}^{s}, d_{j}^{s}\right\}$, one of $c_{j}^{s}$ or $d_{j}^{s}$ has already been placed in $\bar{\alpha}$; if not, $x \in\left\{c_{j}^{s}, d_{j}^{s}\right\}$, in which case the other element of $\left\{c_{j}^{s}, d_{j}^{s}\right\}$ is now placed in $\bar{\alpha}$. For each $j$, in order, for which $\left\{c_{j}^{s}, d_{j}^{s}\right\} \cap\left\{x, \varphi_{e}(x)\right\} \neq \varnothing$, let $y$ be the smallest number bigger than $j+1$ such that both it and its successor are free, and define $c_{j}^{s+1}=y$ and $d_{j}^{s+1}=y+1$.

Let each marker which had an assignment at the end of stage $s$, and which has not been given a new assignment by the above instructions, keep its assignment through stage $s+1$. Put both $c_{b_{s}}^{s+1}$ and $d_{b_{s}}^{s+1}$ into $\bar{\alpha}$. Define $p_{s+1}^{s+1}=0$ and let $i_{s+1}^{s+1}$ be the least free number in $I_{s+1}$.

We begin our analysis with some simple observations. First, $\alpha=N-\bar{\alpha}$ is co-recursively enumerable. Since each marker moves finitely often, each marker eventually stabilizes. For each $s$ and $j, j+1<c_{j}^{s}=d_{j}^{s}-1$. Finally, since $i_{j} \in \alpha \cap I_{j}, \bar{\alpha}$ is dense.

We now show that if $y$ is newly enumerated in $\bar{\alpha}$ at stage $s+1$, then $y \geqq b_{s}+1$, with equality only in certain specified circumstances. Suppose first that $e$ required attention and was given that attention using $x$ at stage $s+1$. If $y=\varphi_{e}(x)$, then we have $b_{s}+1 \leqq x<$ $\varphi_{e}(x)$ so that $y>b_{s}+1$. If $y \in\left\{c_{j}^{s}, d_{j}^{s}\right\}$ and $y$ is placed in $\bar{\alpha}^{s+1}$ because $\left\{c_{j}^{s}, d_{j}^{s}\right\} \cap\left\{x, \varphi_{e}(x)\right\} \neq \varnothing$, then, besides the case $y=\varphi_{e}(x)$ treated above, we must consider two cases: If $y=d_{j}^{s}$, then $c_{j}^{s}=x \geqq b_{s}+1$, so that $y=d_{j}^{s}=c_{j}^{s}+1>b_{s}+1$; if $y=c_{j}^{s}$, then $d_{j}^{s}=x$, so that $x>b_{s}+1$, (since $x=b_{s}+1$ only if $x=c_{j^{\prime}}^{s}$ for some $j^{\prime}$ and no $c_{j^{\prime}}^{s}$ can be $d_{j}^{s}$ ), and hence $y=x-1 \geqq b_{s}+1$. Whether any $e$ was given attention or not, the only other elements newly enumerated in $\bar{\alpha}$ at stage $s+1$ are $c_{b_{s}}^{s+1}$ and $d_{b_{s}}^{s+1}$. But then by the next to the last observation of the preceding paragraph, $b_{s}+1<c_{b_{s}}^{s+1}<d_{b_{s}}^{s+1}$. Thus, in all cases, $y \geqq b_{s}+1$. Note that $y=b_{s}+1$ only when $e$ requires attention at stage $s+1$ 
and is given attention using $x=d_{j}^{s}$ where $y=c_{j}^{s}$ and $x=b_{s}+2$.

Now we verify (C) by showing that its failure would imply that $\beta$ is recursive. Actually, we shall prove much more, namely, that if $\phi$ is any partial recursive function which maps $\alpha$ into $\alpha$, then there can be no infinite subset $\gamma$ of $\alpha$ such that $\varphi$ maps $\gamma$ into $\gamma$ in a 1-1 fashion and such that $\varphi(x) \neq x$ for all $x \in \gamma$. (This implies (C); becase if $\alpha \leqq_{c}^{\varphi} \alpha$ but $\varphi(x) \neq x$ for some $x \in \alpha$, then

$$
\gamma=\left\{\varphi^{n}(x) \mid n \in N\right\}
$$

would violate the above.) Suppose $\varphi=\varphi_{a}$ with $\gamma$ is a counterexample. Choose $s_{0}$ so large that $\left\{p_{j}, i_{j}, c_{j}, d_{j} \mid j<a\right\}=M_{a}$ and $p_{a}$ have all stabilized by stage $s_{0}$; thus for $s>s_{0}, M_{a}^{s}=M_{a}$. Note that $p_{a}=0$ since $p_{a}>0$ implies $p_{a} \in \alpha$ and $\varphi\left(p_{a}\right) \in \bar{\alpha}$, contradicting the assumption that $\varphi$ maps $\alpha$ into $\alpha$.

Next observe that there are infinitely many $x \in \gamma$ such that $x<$ $\varphi(x)$ and $\{x, \varphi(x)\} \cap M_{a}=\varnothing$. To see this, first note that if $f$ is a 1-1 function mapping an infinite subset $M$ of $N$ into itself and has no fixed point, then there is an infinite subset $M_{1} \subseteq M$ such that $m<f(m)$ for all $m \in M_{1}$. Now let $M$ be $\gamma, f$ be $\varphi$, and use the finiteness of $M_{a}$.

In view of this, given any $z$ we can effectively find a $y>z+1$ and an $s_{1}>s_{0}$ such that $\varphi^{s_{1}}(y)$ is defined, $y<\varphi^{s_{1}}(y), y \notin \bar{\alpha}^{s_{1}} \cup M_{a}$ and $\varphi(y) \notin M_{a}$. We shall see below that if $s \geqq s_{1}$ then $b_{s}>z$. Hence $z \in \beta$ iff $z \in\left\{b_{0}, b_{1}, \cdots, b_{s_{1}}\right\}$. Since $s_{1}$ was found recursively from $z$, this implies that $\beta$ is recursive.

To show that if $s \geqq s_{1}$ then $b_{s}>z$, it suffices to show that $y \notin \bar{\alpha}^{s}$ for any $s \geqq s_{1}$. For if that were so, then, since $M_{a}^{s}=M_{a}$ for all $s \geqq s_{1}$, the only reason that $a$ does not require attention at stage $s+1$ is that $b_{s}+1 \geqq y$. But since $y>z+1$, we get $b_{s}>z$ for all $s \geqq s_{1}$.

We proceed by induction on $s \geqq s_{1}$. So assume $y \notin \bar{\alpha}^{s}$. If $y$ were put into $\bar{\alpha}$ at stage $s+1$, then $y \geqq b_{s}+1$. If $y>b_{s}+1$ then $a$ would be given attention using $y$ at stage $s+1$; if $y=b_{s}+1$ then some $e>a$ was given attention using $x=b_{s}+2=d_{j}^{s}$ at stage $s+1$ -but then $y=c_{j}^{s}=b_{s}+1$ so that instead $a$ would have been given attention using $y$ at stage $s+1$. Hence $y \notin \alpha^{s+1}$. This completes the proof of $(\mathrm{C})$ and its generalization.

As to (A), we have already noted that $a$ is dense. To show that $\bar{\alpha}$ is also dense, given any rational interval $I_{j}$ we select a recursive function $\phi$ which is the identity outside of $I_{j}$ and is strictly increasing within $I_{j}$. Since $\alpha$ is dense, there is an $x \in \alpha \cap I_{j}$. Since $x \otimes \varphi(x)$, it follows from (C) that $\varphi$ cannot map $\alpha$ into $\alpha$. So for some $y \in \alpha, \varphi(y) \notin \alpha$. In particular, $\varphi(y) \neq y$ so $\varphi(y) \in I_{j}$. Thus $\varphi(y) \in$ $\bar{\alpha} \cap I_{j}$ so that $\bar{\alpha}$ is dense and (A) is proved.

That $\alpha \leqq_{T} \beta$ follow from our observation that if $y$ is first enu- 
merated in $\bar{\alpha}$ at stage $s+1$, then $y \geqq b_{s}+1$. To see whether or not $y \in \alpha$, find $s$ recursively in $\beta$ such that for $s^{\prime} \geqq s$ we have $b_{s^{\prime}}>y$. Then $y \in \alpha$ iff $y \notin \bar{\alpha}^{s+1}$.

To see that $\beta \leqq_{T} \alpha$, first observe that $j \in \beta$ iff $c_{j} \in \bar{\alpha}$. The following procedure determines, recursively in $\alpha$, whether or not $j \in \beta$. It $\left\{c_{j}^{0}, d_{j}^{0}\right\} \subseteq \alpha$ then $c_{j}^{0}=c_{j}$ and $j \notin \beta$. Otherwise, look at the construction and find the stage at which $\left\{c_{j}^{0}, d_{j}^{0}\right\} \cap \bar{\alpha}$ became nonempty. (Note that for this part of the argument it was necessary earlier to have put something into $\bar{\alpha}$ whenever the $c_{j}$ marker was moved; this explains the role of the markers $d_{j}$ in the construction.) If the cause of that action was the membership of $j$ in $\beta$ then of course $j \in \beta$. Otherwise $c_{j}$ and $d_{j}$ are given new assignments and we may repeat the analysis. Since $c_{j}$ has a stable assignment, the procedure terminates after a finite number of iterations. This completes the proof of (B).

We observe that the set $\alpha$ constructed above is immune. For the stronger version of (C) proved above implies that if $\gamma$ is any infinite subset of $\alpha$ and $\phi$ is any partial recursive function (not necessarily order-preserving) which maps $\gamma$ into $\gamma$ in a 1-1 fashion, then $\varphi(x)=x$ for some $x \in \gamma$. On the other hand, if $\gamma$ were an infinite recursively enumerable subset of $\alpha$, then there would clearly be partial recursive functions mapping $\gamma$ into $\gamma$ with no fixed points.

It should be noted also that Theorem 1 has a purely set-theoretic counterpart. That is, the generalization of $(C)$ proved above yields the following corollary.

COROLLARY. Let $\beta$ be any recursively enumerable set which is not recursive. There exists a co-recursively enumerable set $\alpha$ of natural numbers such that (i) $\alpha$ is Turing equivalent to $\beta$, and (ii) if $\phi$ is any partial recursive function mapping $\alpha$ into $\alpha$, then $\varphi(\alpha)=a$ for all but a finite number of $a \in \alpha$.

3. Recursive analogues of some results of Dushnik and Miller and Sierpiński. Theorem 2 , as quoted in $\S 1$, is an immediate consequence of Theorem 1. The following lemma will be useful in proving Theorems 3,4 and 5.

LEMMA 1. Let $\beta$ be any recursively enumerable set which is not recursive. Let $\alpha$ be any co-recursively enumerable set satisfying the conditions of Theorem 1. Let $c \notin \alpha$ and $d \in \alpha$. Then both $\alpha \cup$ $\{c\}$ and $\alpha-\{d\}$ satisfy the conditions of Theorem 1 .

Proof. In both cases, only (C) needs attention since the other conditions are immediate. Consider first $\delta=\alpha-\{d\}$ and suppose that 
$\delta \leqq_{c}^{\varphi} \delta$. There are three possibilities:

(i) for some $d^{\prime} \in \delta, d^{\prime} \otimes d$ and $\varphi\left(d^{\prime}\right) \otimes d$;

(ii) for some $d^{\prime} \in \delta, d^{\prime} \ominus d$ and $\varphi\left(d^{\prime}\right) \oslash d$;

(iii) for all $d^{\prime} \in \delta$, both $d^{\prime} \oslash d$ implies $\varphi\left(d^{\prime}\right) \oslash d$ and $d^{\prime} \ominus d$ implies $\varphi\left(d^{\prime}\right) \oslash d$.

We shall see that (i), and similarly (ii), contradicts (C) for $\alpha$, while (iii) implies that $\phi$ is the identity on $\delta$.

Let $d^{\prime}$ satisfy (i). Define

$$
\psi(x) \cong\left\{\begin{array}{l}
\varphi(x) \text { if } x \ominus d \text { and } \varphi(x) \text { is defined ; } \\
x \text { if } x \triangleq d .
\end{array}\right.
$$

Then $\psi$ is a partial recursive function whose domain includes $\delta U$ $\{d\}=\alpha$ and $\alpha \leqq_{0}^{\psi} \alpha$. Now let $d^{*} \in \alpha$ be such that $\varphi\left(d^{\prime}\right) \oslash d^{*} \ominus d \ominus d^{\prime}$. Since $\varphi$ is order preserving on $\delta$ and $d^{*} \in \delta$, we have $\varphi\left(d^{*}\right) \diamond \varphi\left(d^{\prime}\right) \diamond$ $d^{*}$. But then $d^{*} \in \alpha$ and $\varphi\left(d^{*}\right) \oslash d^{*}$, contradicting (C) for $\alpha$. The contradiction in case (ii) is deduced symmetrically. Finally, in case (iii), define

$$
\psi(x) \cong\left\{\begin{array}{lll}
\varphi(x) & \text { if } & x \neq d \\
d & \text { if } & x=d
\end{array}\right.
$$

Then $\alpha \leqq_{c}^{\psi} \alpha$ so that $\psi(\alpha)=\alpha$ for all $a \in \alpha$. Hence $\varphi(\alpha)=a$ for all $a \in \delta$.

The proof that $\gamma=\alpha \cup\{c\}$ satisfies condition (C) is similar.

For the remainder of this section, let $\beta$ be any recursively enumerable set which is not recursive and let $\alpha$ be any co-recursively enumerable set satisfying the conditions of Theorem 1 for $\beta$. Let $\alpha=\left\{a_{0}, a_{1}, a_{2}, \cdots\right\}$ and $\bar{\alpha}=\left\{c_{0}, c_{1}, c_{2}, \cdots\right\}$ where $i \neq j$ implies $a_{i} \neq \alpha_{j}$ and $c_{i} \neq c_{j}$.

Proof of Theorem 3. Let $\gamma_{0}=\alpha$ and $\gamma_{i+1}=\gamma_{i} \cup\left\{c_{i}\right\}$. The identity function is a recursive embedding of $\gamma_{i}$ into $\gamma_{i+1}$. Lemma 1 shows that each $\gamma_{i}$ satisfies the conditions placed on $\alpha$ in Theorem 1 . If $\gamma_{i+1} \leqq_{c}^{\varphi} \gamma_{i}$, then also $\gamma_{i+1} \leqq_{c}^{\varphi} \gamma_{i+1}$ since $\gamma_{i} \subseteq \gamma_{i+1}$. Since $\gamma_{i+1}$ satisfies condition (C), $\varphi(x)=x$ for all $x \in \gamma_{i+1}$. In particular, $\varphi\left(c_{i}\right)=c_{i} \notin \gamma_{i}$ so $\gamma_{i+1} \bigsqcup_{c} \gamma_{i}$. Notice that this implies that $\gamma_{i} \leqq_{c} \gamma_{j}$ iff $i \leqq j$.

Proof of Theorem 4. Let $\delta_{0}=\alpha$ and $\delta_{i+1}=\delta_{i}-\left\{a_{i}\right\}$. The identity function is a recursive embedding of $\delta_{i+1}$ into $\delta_{i}$. The remainder of the proof is virtually identical to that of Theorem 3 including the final observation that $\delta_{i} \leqq_{c} \delta_{j}$ iff $i \geqq j$.

Proof of Theorem 5. Let $\eta_{i}=\alpha-\left\{\alpha_{i}\right\}$. Suppose $i \neq j$ and 
$\eta_{i} \leqq_{c}^{\varphi} \eta_{j}$. There are three cases, as in the proof of Lemma 1 above. If for some $d^{\prime} \in \eta_{i}$, both $d^{\prime} \oslash a_{i}$ and $\varphi\left(d^{\prime}\right) \otimes a_{j}$, then we define $\psi$ by

$$
\psi(x) \cong\left\{\begin{array}{lll}
\varphi(x) & \text { if } & x \otimes a_{i} \\
a_{j} & \text { if } & x=a_{i} \\
x & \text { if } & x \otimes a_{i}
\end{array}\right.
$$

and obtain a contradiction. If for some $d^{\prime} \in \eta_{i}$, both $d^{\prime} \ominus a_{i}$ and $\varphi\left(d^{\prime}\right) \oslash a_{j}$, then we define $\psi$ similarly, and obtain a similar contradiction. Finally, if $d^{\prime} \oslash a_{i}$ implies $\varphi\left(d^{\prime}\right) \oslash a_{j}$ and $d^{\prime} \ominus a_{i}$ implies $\varphi\left(d^{\prime}\right) \ominus a_{j}$, we can extend $\varphi$ by mapping $a_{i}$ to $a_{j}$, and again get a contradiction.

Notice that if $i \neq j, \eta_{i}$ and $\eta_{j}$ differ by exactly two elements. This gives us a recursive analogue of Theorem 5 of Sierpiński's paper. Theorems 3, 4 and 5 are analogues of Sierpiński's Theorems 3,4 and 5. Finally, the next theorem is an analogue of his Theorem 6 .

THEOREM 6. There exist co-recursively enumerable sets $\alpha_{0}$ and $\alpha_{1}$, each of which is Turing equivalent to $\beta$ and bi-dense, satisfying the following conditions: $\alpha_{0} \leqq_{c} \alpha_{1}, \alpha_{1} \varliminf_{c} \alpha_{0}$, and if $\alpha_{0} \leqq_{c} \alpha_{2} \leqq_{c} \alpha_{1}$ then either $\alpha_{2} \leqq_{c} \alpha_{0}$ or $\alpha_{1} \leqq_{c} \alpha_{2}$.

Proof. Let $\alpha_{0}=\alpha$ and $\alpha_{1}=\alpha \cup\left\{c_{0}\right\}=\gamma_{1}$. By Theorems 3 only the last assertion still requires proof. Suppose then $\alpha_{0} \leqq_{c}^{\varphi_{0}} \alpha_{2}$ and $\alpha_{2} \leqq_{c}^{\varphi_{1}} \alpha_{1}$. Defining $\varphi=\varphi_{1} \cdot \varphi_{0}$ we see $\alpha_{0} \leqq_{c}^{\varphi} \alpha_{1}$ We now show this can be strengthened to $\alpha_{0} \leqq_{c}^{\varphi} \alpha_{0}$. Suppose not and let $\varphi\left(a^{*}\right)=c_{0}$ where $a^{*} \in a_{0}$. We may assume $a^{*} \oslash c_{0}$ without loss of generality. Let $\varphi^{*}$ be defined by the conditions:

$$
\varphi^{*}(x) \cong\left\{\begin{array}{lll}
x & \text { if } & x \triangleq a^{*} \\
\varphi(x) & \text { if } & x \ominus a^{*} \text { and } \varphi(x) \text { is defined } .
\end{array}\right.
$$

$\alpha<_{c}^{\varphi^{*}} \alpha$ so $\varphi^{*}(\alpha)=\alpha$ for all $\alpha \in \alpha$. In particular for $c_{0} \ominus \alpha \ominus a^{*}$ and $a \in \alpha, \varphi(a) \ominus \varphi\left(a^{*}\right)=c_{0} \otimes a=\varphi^{*}(a)=\varphi(a)$. This contradiction shows $\alpha_{0} \leqq_{0}^{\varphi} \alpha_{0}$. It follows that $\varphi(a)=a$ for all $a \in \alpha$.

If $\varphi_{1}(x) \neq c_{0}$ for all $x \in \alpha_{2}$, then $\alpha_{2} \leqq_{c}^{\varphi_{1}} \alpha_{0}$, since $\alpha_{1}=\alpha_{0} \cup\left\{c_{0}\right\}$. Otherwise let $\varphi_{1}(d)=c_{0}$. Since $\varphi_{1} \cdot \varphi_{0}$ is the identity on $\alpha_{0}$, it follows that $\varphi_{1}$ maps $\alpha_{2}-\{d\}$ onto $\alpha_{1}-\left\{c_{0}\right\}$. Furthermore, if $\varphi_{1}(a)=b$ for $a \in \alpha_{2}-\{d\}$, then $\varphi_{0}(b)=a$. So we can define a partial recursive map $\psi$ by $\psi(b) \cong\left\{\begin{array}{ll}\varphi_{0}(b) & \text { if } b \neq c_{0} \\ d & \text { if } b=c_{0},\end{array}\right.$ and it is easily verified that $\alpha_{1} \leqq_{c}^{\psi} \alpha_{2}$. This comples the proof.

4. Further results. The following lemma will be needed in the proof of Theorem 7 . 
LEMma 2. If $\alpha_{1}$ and $\alpha_{2}$ are two subsets of $Q$, let $\alpha_{1} \oplus \alpha_{2}=$ $f\left(\alpha_{1}\right) \cup g\left(\alpha_{2}\right)$ where $f$ and $g$ are fixed recursive order-preserving functions of $Q$ onto $(-\infty, 0)$ and $(0,+\infty)$ respectively.

(i ) $\alpha_{1}$ and $\alpha_{2}$ bi-dense implies $\alpha_{1} \oplus \alpha_{2}$ bi-dense.

(ii) $\quad \alpha_{1}$ and $\alpha_{2}$ co-recursively enumerable implies $\alpha_{1} \oplus \alpha_{2}$ co-recursively enumerable.

(iii) $\operatorname{deg}\left(\alpha_{1} \oplus \alpha_{2}\right)=\operatorname{deg} \alpha_{1} \cup \operatorname{deg} \alpha_{2}$.

(iv) $\alpha_{1}$ and $\alpha_{2}$ satisfying condition (C) implies that $\alpha_{1} \oplus \alpha_{2}$ satisfies condition (C).

Proof. (i) and (ii) are obvious. Since complete information about membership in $\alpha_{1} \oplus \alpha_{2}$ is equivalent to complete information about membership in both $\alpha_{1}$ and $\alpha_{2}$, (iii) is correct. As for (iv), if $\alpha_{1} \oplus$ $\alpha_{2} \leqq_{c}^{\varphi} \alpha_{1} \oplus \alpha_{2}$, then, as in Lemma 1 and subsequent arguments, there are three cases, the first two leading to contradictions and the third leading to the conclusion that $\varphi$ is the identity on $\alpha_{1} \oplus \alpha_{2}$

THEOREM 7. Let $\beta$ be any recursively enumerable set which is not recursive. Then there exists a collection $\left\{\gamma_{q} \mid q \in Q\right\}$ of co-recursively enumerable subsets of $Q$ satisfying conditions (A), (B) and (C) of Theorem 1 and such that for each $q, q^{\prime} \in Q$

$$
\gamma_{q} \leqq_{c} \gamma_{q^{\prime}} \quad \text { iff } \quad q \supseteqq q^{\prime} \text {. }
$$

Proof. Let $R$ be a fixed recursive subset of $Q$ satisfying the following properties: The order type of $R$ is $2 \cdot \eta$. $R$ is the disjoint union of two recursive sets $R_{p}$ and $R_{s}$. There is a recursive function which assigns to each $r \in R_{p}$ that element $r^{+} \in R_{s}$ which is its immediate successor in $R$. Every element of $R_{s}$ is the immediate successor in $R$ of some $r \in R_{p}$. Note that $R_{p}$ is order isomorphic to $Q$.

Let $\alpha$ be the set constructed in Theorem 1 and recall, as discussed immediately after its proof, that $\alpha$ is also immune. For each $r \in R_{p}$, let $\alpha_{r}=\alpha-\left\{(\mu x)\left(x \in\left(s, s^{+}\right) \cap \alpha\right) \mid s \in R_{p}, s \otimes r\right\}$. This is well defined since $\alpha$ is dense. For each $r \in R_{p}, \alpha_{r}$ is recursive in $\alpha, \alpha_{r}$ is bi-dense, and $\alpha_{r}$ is immune. To see that $\alpha_{r}$ is co-recursively enumerable, consider the following procedure, which gives a recursive enumeration of $\bar{\alpha}_{r}$. Enumerate $\bar{\alpha}$ into $\bar{\alpha}_{r}$ and simultaneously enumerate $\left\{s \in R_{p} \mid s \oslash r\right\}$; when $s$ is enumerated, find the least $x$ in $\left(s, s^{+}\right)$which is not yet in $\bar{\alpha}$ and put it in $\bar{\alpha}_{r}$ - if that $x$ eventually turns up in the enumeration of $\bar{\alpha}$, then a new $x$ in $\left(s, s^{+}\right)$can be selected for $\bar{\alpha}_{r}$; since $\alpha$ is dense, eventually the $x$ selected and put into $\bar{\alpha}_{r}$ will not also appear in $\bar{\alpha}$. Note that, by the uniformity of these definitions, $\alpha_{r} \subseteq \alpha_{s}$ iff $r \ominus s$.

For each $r \in R_{p}$ let $\gamma_{r}=\alpha \oplus \alpha_{r}$. Then, by Lemma 2, each $\gamma_{r}$ is 
bi-dense, co-recursively enumerable, and is Turing-equivalent to $\alpha$ (since $\alpha_{r}$ is recursive in $\alpha$ ) and hence to $\beta$. To see that $\gamma_{r}$ satisfies (C) it suffices, by Lemma 2, to verify that $\alpha_{r}$ satisfies (C). But if $\alpha_{r} \leqq_{c}^{\varphi} \alpha_{r}$ and for some $a \in \alpha_{r}, \varphi(a) \neq \alpha$, then, assuming $\varphi(\alpha) \oslash \alpha$ we get an infinite recursively enumerable subset $\alpha, \varphi(\alpha), \varphi^{2}(\alpha), \phi^{3}(\alpha), \cdots$ of $\alpha_{r}$, contradicting the fact that it is immune.

Finally, we must verify that if $r, s \in R_{p}$ then $\gamma_{r} \leqq_{c} \gamma_{s}$ iff $r \triangleq s$. If $r \otimes s$ then $\gamma_{r} \leqq_{c}^{\text {id }} \gamma_{s}$, so clearly $\gamma_{r} \leqq_{c} \gamma_{s}$. If, on the other hand, $\gamma_{r} \leqq_{c}^{\varphi} \gamma_{s}$, but $s \bigotimes r$, then, since $\gamma_{s} \leqq_{c}^{\text {id }} \gamma_{r}$ we get $\gamma_{r} \leqq_{c}^{\varphi} \gamma_{r}$. Hence $\phi$ is the identity on $\gamma_{r}$, so that $\gamma_{r} \subseteq \gamma_{s}$ which implies that $\alpha_{r} \subseteq \alpha_{s}$ and thus $r \triangleq s$. Hence $\gamma_{r} \leqq_{c} \gamma_{s}$ iff $r \triangleq s$.

Since $R_{p}$ is order-isomorphic to $Q$, this completes the proof.

COROLlaRY. If $L$ is any countable linear ordering, there is a collection of co-recursively enumerable subsets of $Q$ satisfying conditions (A), (B) and (C) of Theorem 1 which, with respect to $\leqq_{c}$, is order-isomorphic to $L$.

The proof of Theorem 8 follows easily from the following lemma.

LEMMA 3. If $\alpha_{1}$ and $\alpha_{2}$ are both bi-dense and immune and if $\alpha_{2}-\alpha_{1}$ is finite, $\alpha_{1} \leqq_{c} \alpha_{2}$ iff $\alpha_{1} \leqq \alpha_{2}$.

Proof. One direction is trivial. Suppose, for the other direction, that $\alpha_{1} \leqq{ }_{c}^{\varphi} \alpha_{2}$ but that $\alpha_{1} \nsubseteq \alpha_{2}$. Let $\alpha_{0} \in \alpha_{1}-\alpha_{2}$. Then $\varphi\left(a_{0}\right) \in \alpha_{2}$ so $\varphi\left(a_{0}\right) \neq a_{0}$. Assume without loss of generality that $\varphi\left(a_{0}\right)<a_{0}$. Then either

$$
a_{0} \oslash \varphi\left(a_{0}\right) \oslash \varphi^{2}\left(a_{0}\right)>\cdots
$$

and all of these are in $\alpha_{1} \cap \alpha_{2}$, or there is a $k$ for which $\varphi^{k-1}\left(a_{0}\right) \epsilon$ $\alpha_{1} \cap \alpha_{2}$, but $\phi^{k}\left(\alpha_{0}\right) \in \alpha_{2}-\alpha_{1}$. Since the former would give us an infinite recursively enumerable subset of $\alpha_{1}$, contradicting its immunity, there must be such a $k$. Now $\phi^{k}\left(a_{0}\right) \oslash \phi^{k-1}\left(a_{0}\right)$ and $\alpha_{1}$ is dense so that there is an $a_{1} \in \alpha_{1}$ with $\phi^{k}\left(a_{0}\right) \ominus \alpha_{1} \ominus \phi^{k-1}\left(a_{0}\right)$. Since $a_{1} \in \alpha_{1}$ and $\varphi^{k-1}\left(a_{0}\right) \in \alpha_{1}$ and $\varphi$ is order-preserving on $\alpha_{1}$, it follows that $\varphi\left(a_{1}\right) \oslash$ $\phi^{k}\left(a_{0}\right)=x_{0}$. Using $a_{1}$ in place of $\alpha_{0}$ we may repeat the above argument to find another $k$ such that $\phi^{k-1}\left(a_{1}\right) \in \alpha_{1} \cap \alpha_{2}$ but $x_{1}=\phi^{k}\left(a_{1}\right) \in \alpha_{2}-\alpha_{1}$. Also, $x_{1}=\varphi^{k}\left(a_{1}\right) \otimes \varphi\left(a_{1}\right)=x_{0}$ so that $x_{1} \ominus x_{0}$. Since $x_{1} \otimes \phi^{k-1}\left(a_{1}\right)$ we can choose $a_{2} \in \alpha_{1}$ such that $x_{1} \ominus \alpha_{2} \otimes \phi^{k-1}\left(\alpha_{1}\right)$ and continue the argument forever generating an infinite sequence $\cdots \otimes x_{2} \otimes x_{1} \otimes x_{0}$ of elements of $\alpha_{2}-\alpha_{1}$, contradicting its finiteness. Hence if $\alpha_{1} \leqq_{c} \alpha_{2}$ it follows that $\alpha_{1} \subseteq \alpha_{2}$.

THEOREM 8. Let $\beta$ be any recursively enumerable set which is 
not recursive and let $C$ be any finite partial ordering. Then there is a collection of co-recursively enumerable sets, each bi-dense in $Q$, each Turing-equivalent to $\beta$, such that, under $\leqq_{c}$, this collection is order-isomorphic to $C$.

Proof. Let $(C, \sqsubseteq)$ be a finite partial ordering with $n$ elements. Let $c_{1}, \cdots, c_{n}$ be $n$ elements of $\bar{\alpha}$ where $\alpha$ is the set constructed in Theorem 1. Let $f$ be a 1-1 correspondence between $C$ and $\left\{c_{1}, \cdots, c_{n}\right\}$. Let $\alpha_{x}=\alpha \cup\left\{c_{f(y)} \mid y \equiv x\right\}$ for each $x \in C$. Clearly, if $x$ and $y$ are in $C$, then $\alpha_{y}-\alpha_{x}$ is finite, so that, by Lemma $3, \alpha_{x} \leqq_{c} \alpha_{y}$ iff $\alpha_{x} \leqq \alpha_{y}$. But $\alpha_{x} \leqq \alpha_{y}$ iff $x \sqsubseteq y$, and hence $\alpha_{x} \leqq{ }_{c} \alpha_{y}$ iff $x \sqsubseteq y$, as was to be proved.

5. Concluding remarks. We view the results of this note in at least two contexts. One is to reveal the strong analogy between uncountable suborderings of the reals and non-recursive suborderings of the rationals. The examples of co-recursively enumerable suborderings of the rationals suggests that they be considered under $\leqq_{c}$ as a possible legitimate effectivization of uncountable suborderings of the reals under $\leqq$. This, in turn, may lead to the study of large classes of suborderings of the rationals under $\leqq_{c}$ and to the general theories of $\leqq_{c}$ and $\leqq$.

Our results are also part of a collection of attempts to analyze the effectiveness or computational content of some infinitary combinatorial theorems. Theorem 1 shows that Dushnik and Miller's proof of Theorem II was, from a recursion theoretic point of view, best possible. That is, their proof provides a co-recursively enumerable construction while there is no recursive construction. Although we did not deal with their proof of Theorem I at any length in this note, we did mention that certain effective versions of it are false. In fact, there is a recursive subset of the rationals whose classical order type is $\omega$ which is not recursively similar to any proper subset of itself.

\section{REFERENCES}

1. Ben Dushnik and E. W. Miller, Concerning similarity transformations of linearly ordered sets, Bull. Amer. Math. Soc., 46 (1940), 322-326.

2. Richard Laver, On Fraïsé's order type conjecture, Annals of Math., 93 (1971), 89111.

3. Hartley Rogers, Jr., Theory of Recursive Functions and Effective Computability, McGraw-Hill, 1967, xix + 482 pp.

4. Warcław Sierpiński, Sur les types d'ordre des ensembles lineaires, Fundamenta Mathematicae, 37 (1950), 253-264.

Received June 8, 1976. The research reported here was partially supported by the National Science Foundation grants GP 38876 and GP 28348. 
70 LOUISE HAY, ALFRED B. MANASTER AND JOSEPH G. ROSENSTEIN

University of Illinois at Chicago Circle

University of California at SAN Diego

Rutgers University, New Brunswick, New Jersey, New Brunswick, NJ 08903 and Institute for Advanced Study, Princeton, New Jersey 


\section{PACIFIC JOURNAL OF MATHEMATICS}

\section{EDITORS}

RICHARD ARENS (Managing Editor)

University of California

Los Angeles, California 90024

C. W. CURTIS

University of Oregon

Eugene, OR 97403

C. C. MOORE

University of California

Berkeley, CA 94720

\section{J. DugundJI}

Department of Mathematics University of Southern Californıa Los Angeles, California 90007

R. FinN AND J. Milgram Stanford University Stanford, California 94305

\section{ASSOCIATE EDITORS}

E. F. BECKENBACH

B. H. NEUMANN

F. WOLF

K. YOSHIDA

\section{SUPPORTING INSTITUTIONS}

UNIVERSITY OF BRITISH COLUMBIA CALIFORNIA INSTITUTE OF TECHNOLOGY UNIVERSITY OF CALIFORNIA MONTANA STATE UNIVERSITY UNIVERSITY OF NEVADA, RENO NEW MEXICO STATE UNIVERSITY OREGON STATE UNIVERSITY UNIVERSITY OF OREGON OSAKA UNIVERSITY
UNIVERSITY OF SOUTHERN CALIFORNIA STANFORD UNIVERSITY UNIVERSITY OF TOKYO UNIVERSITY OF UTAH WASHINGTON STATE UNIVERSITY UNIVERSITY OF WASHINGTON AMERICAN MATHEMATICAL SOCIETY NAVAL WEAPONS CENTER 


\section{Pacific Journal of Mathematics}

\section{Vol. 71, No. $1 \quad$ November, 1977}

Charalambos D. Aliprantis and Owen Sidney Burkinshaw, On universally complete Riesz spaces ............................. 1

Stephen Richard Bernfeld and Jagdish Chandra, Minimal and maximal solutions of nonlinear boundary value problems .................

John H. E. Cohn, The length of the period of the simple continued fraction of

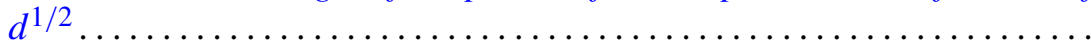

Earl Vern Dudley, Sidon sets associated with a closed subset of a compact abelian group .................................... 33

Larry Finkelstein, Finite groups with a standard component of type $J_{4} \ldots \ldots$

Louise Hay, Alfred Berry Manaster and Joseph Goeffrey Rosenstein, Concerning partial recursive similarity transformations of linearly ordered sets .......................................

Richard Michael Kane, On loop spaces without $p$ torsion. II ............

William A. Kirk and Rainald Schoneberg, Some results on

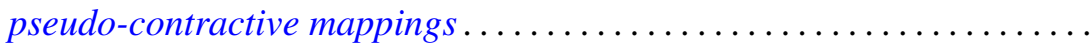

Philip A. Leonard and Kenneth S. Williams, The quadratic and quartic character of certain quadratic units. I. .

Lawrence Carlton Moore, A comparison of the relative uniform topology and the norm topology in a normed Riesz space .................

Mario Petrich, Maximal submonoids of the translational hull 119

Mark Bernard Ramras, Constructing new R-sequences . . .

Dave Riffelmacher, Multiplication alteration and related rigidity properties

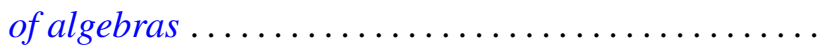

Jan Rosiński and Wojbor Woyczynski, Weakly orthogonally additive functionals, white noise integrals and linear Gaussian stochastic processes.

Ryōtarō Satō, Invariant measures for ergodic semigroups of operators

Peter John Slater and William Yslas Vélez, Permutations of the positive integers with restrictions on the sequence of differences...

Edith Twining Stevenson, Integral representations of algebraic cohomology classes on hypersurfaces ........................

Laif Swanson, Generators of factors of Bernoulli shifts . 